\title{
Diagnóstico diferencial entre el espectro autista y el espectro esquizofrénico
}

\author{
Isabel Paula-Pérez
}

Introducción. La distinción nosológica entre espectro autista y espectro esquizofrénico está hoy en día claramente delimitada, a pesar de las evidencias científicas de la relación genética entre ambas condiciones. El solapamiento de síntomas negativos de la esquizofrenia con ciertas manifestaciones autistas y la atribución errónea de síntomas positivos de la esquizofrenia en el autismo por profesionales no familiarizados con los trastornos del espectro autista ponen de relieve la importancia de descifrar las claves que permitan el diagnóstico diferencial, o la valoración de la comorbilidad y coocurrencia entre ambos espectros cuando así sea.

Desarrollo. El artículo analiza y desgrana las manifestaciones del autismo que pudieran ser erróneamente confundidas con la dimensión psicótica y la dimensión de desorganización correspondientes a los síntomas positivos del espectro de la esquizofrenia, así como esclarecer las explicaciones psicológicas que justifican la manifestación de ciertos síntomas negativos asociados con frecuencia al autismo.

Conclusión. Las claves para determinar si las manifestaciones clínicas son propias del espectro autista, del espectro esquizofrénico o fruto de la comorbilidad radican en la valoración de la historia de desarrollo de la persona, el pródromo e inicio de la alteración, su evolución y la presencia o ausencia de síntomas positivos de la esquizofrenia. Su determinación será crucial para ayudar al profesional en la toma de decisiones tanto diagnóstica como de tratamiento.

Palabras clave. Comorbilidad. Diagnóstico diferencial. Espectro autista. Espectro esquizofrénico. Nosología. Síntomas positivos y negativos.

\section{Introducción}

El diagnóstico diferencial en el ámbito de la salud mental es el procedimiento que nos permite identificar una determinada condición nosológica dentro de las principales clasificaciones con que contamos hoy día -el Manual diagnóstico y estadístico de los trastornos mentales (DSM) de la Asociación Americana de Psiquiatría y la Clasificación Internacional de Enfermedades (CIE) de la Organización Mundial de la Salud-, excluyendo otras posibles entidades que presenten un cuadro clínico semejante al que padece el paciente. Sin embargo, en psicopatología es común la comorbilidad o coocurrencia de dos o más diagnósticos clínicos. Decidir durante el proceso diagnóstico si estamos hablando de trastornos comórbidos o debemos establecer parámetros para el diagnóstico diferencial es uno de los grandes retos de la psicopatología por la importancia que tiene para la comprensión de lo que sucede al paciente y para la elección de un tratamiento $\mathrm{u}$ otro.

Aunque la comunidad científica apoya la distinción entre el autismo y la esquizofrenia u otros trastornos psicóticos, su diagnóstico diferencial o su comorbilidad no siempre son tan obvios en la prác- tica clínica. El aumento y precisión del diagnóstico de trastornos del espectro autista (TEA) ha evidenciado el sobrediagnóstico de esquizofrenia, especialmente en pacientes con un buen funcionamiento en el área intelectual y del lenguaje. La sintomatología de los TEA y algunos de los comportamientos no psicóticos de la esquizofrenia -básicamente el aplanamiento afectivo o la falta de motivaciónno son siempre fácilmente distinguibles [1,2], y también pueden malinterpretarse los pensamientos, ideas $\mathrm{y}$ atribuciones que hacen las personas con TEA, otorgándoles el valor de síntomas positivos de la esquizofrenia, como, por ejemplo, las ideas delirantes.

En ambos espectros se produce una alteración de la interacción social, la comunicación, el procesamiento de las emociones y las funciones ejecutivas. A ello se suma que, en la esquizofrenia de inicio infantil, existe un riesgo significativo de padecer retrasos y alteraciones en el neurodesarrollo anteriores al inicio de la sintomatología psicótica que son similares a los síntomas del autismo. Es lo que denominamos el pródromo de la esquizofrenia. En el estudio de Nicolson et al [3], más del $88 \%$ de los participantes con trastorno psicótico no especificado manifestó un desarrollo social atípico en edades tempranas.
Departamento de Métodos de Investigación y Diagnóstico en Educación. Universitat de Barcelona. Barcelona, España.

Correspondencia:

Dra. Isabel Paula Pérez. Universitat de Barcelona. Campus Mundet. Edif. Llevant, 2. piso, desp. 275. Pg. Vall d'Hebron, 171. E-08035 Barcelona.

E-mail:

isabelpaula@ub.edu

Declaración de intereses: La autora manifiesta la inexistencia de conflictos de interés en relación con este artículo.

Aceptado tras revisión externa: 13.01.12.

Cómo citar este artículo: Paula-Pérez I. Diagnóstico diferencial entre el espectro autista y el espectro esquizofrénico. Rev Neurol 2012; 54 (Supl 1): S51-62.

(c) 2012 Revista de Neurología 
Por ello, resulta necesario orientar a los profesionales de la neuropediatría, la psiquiatría infantojuvenil y la psicología clínica a establecer criterios que ayuden al diagnóstico diferencial y reflexionar sobre aquellas manifestaciones clínicas que pueden resultar confusas a la hora de hacer un diagnóstico de autismo, de esquizofrenia o de determinar su comorbilidad en el caso que así sea. La investigación clínica está haciendo un gran esfuerzo por establecer criterios diagnósticos suplementarios que permitan definir subgrupos de una manera más homogénea, pero las fronteras siguen sin estar siempre claras.

En una primera etapa, el psiquiatra suizo Paul E. Bleuler habló por primera vez en 1911 [4] del autismo como una alteración dentro de la esquizofrenia que implicaba una retirada de la realidad externa. Posteriormente, se sugirió que son dos alteraciones funcional y fenotípicamente distintas [5,6], retirándose el término 'esquizofrenia infantil'. El Journal of Autism and Childhood Schizophrenia -publicado por primera vez en 1971- pasó a llamarse Journal of Autism and Developmental Disorders en 1979. La falta de distinción entre las dos condiciones finalizó en 1980 cuando, por primera vez, la Asociación Americana de Psiquiatría excluyó en el DSM-III los criterios diagnósticos de la esquizofrenia para el autismo, quedando categóricamente separados la esquizofrenia de los trastornos generalizados del desarrollo [7]. Posteriormente, en el año 2000, el DSM-IV-TR reconoció que la esquizofrenia puede desarrollarse en personas con TEA, y que el diagnóstico adicional de esquizofrenia solamente está garantizado si las ideas delirantes y las alucinaciones se presentan por lo menos durante un mes (o menos, en el caso de recibir tratamiento efectivo) y el inicio de los TEA precede claramente al inicio de la esquizofrenia [8].

Lo que no parece tan claro es que los mecanismos patofisiológicos de ambas condiciones estén tan diferenciados. Aunque los estudios epidemiológicos de la relación genética entre el autismo y la esquizofrenia son todavía deficientes, existen evidencias directas e indirectas que confirman que ambas condiciones comparten factores genéticos [9-11]. Recientemente, se han encontrado deleciones en el gen CNTNAP2 en el autismo y la esquizofrenia, aunque existen personas con variante del gen CNTNAP2 que no muestran signos clínicos, lo que sugiere que ambos trastornos son multifactoriales (http://genome.ucsc.edu).

Los últimos estudios de 2011 han hallado que el gen Neurexin-1 (NRXN1), localizado en el cromosoma 2p16.3, es el gen con mayor evidencia de susceptibilidad compartida para el autismo y la esqui- zofrenia [12], específicamente, evidencias muy significativas de deleciones en dicho gen en ambos espectros [13,14]. Dado que el NRXN1 también se ha asociado con el retraso mental, hace pensar que disrupciones en este gen, en especial deleciones, suponen un riesgo para fenotipos relacionados con alternaciones del neurodesarrollo.

La estructura cerebral en el autismo y la esquizofrenia también comparten determinadas similitudes: una menor materia gris en el giro parahipocámpico derecho, corteza cingulada posterior, putamen, clastrum y tálamo izquierdo en ambos trastornos [15]; vulnerabilidad neuronal relacionada con el lóbulo frontal y los circuitos neuronales relacionados con él [16,17]; o hipoactivación de la amígdala durante tareas de matiz emocional, provocando anormalidades en el procesamiento social tanto en los TEA como en la esquizofrenia $[18,19]$. También se ha postulado en la investigación que las estructuras del lóbulo temporal medial desempeñan un papel importante en el autismo y la esquizofrenia; por ejemplo, se considera que el hipocampo actúa como procesador central 'multidimensional', que integra la información motivacional y contextual que permite generar respuestas adaptativas, y su disfunción se traduce en el área del lenguaje, la conducta y la emoción [20].

Los estudios epidemiológicos reflejan que la prevalencia de comorbilidad de la esquizofrenia en la población con TEA es la misma que para los grupos control, del 1\%. Estudios recientes hechos a gran escala confirman que la incidencia de síntomas positivos de la esquizofrenia en personas con autismo no es significativamente más alta que en la población neurotípica [21,22]. Más recientemente, estudios de seguimiento de niños con autismo reconfirman que es muy poco usual que aparezcan síntomas psicóticos en la juventud o en la vida adulta $[23,24]$. Sin embargo, llama la atención que la comorbilidad entre TEA y esquizofrenia aumenta significativamente en unidades de atención psiquiátrica intensiva de adultos [25]. Los datos no son todavía concluyentes.

¿Cómo puede el profesional discernir si las manifestaciones conductuales son propias de una condición, de la otra, o fruto de la comorbilidad? El diagnóstico diferencial y la comorbilidad del espectro autista y del espectro esquizofrénico suponen, en algunos casos, un reto para los clínicos. No obstante, una evaluación adecuada de la presentación cualitativa de los síntomas, además de una presencia o ausencia de criterios diagnósticos clave para cada espectro, pueden dar buena información para la toma de decisiones y para el tratamiento. 


\section{Desarrollo}

Referirnos al autismo y a la esquizofrenia como 'espectros' responde a la propuesta que nos ofrece la futura quinta edición del DSM (DSM-5), pendiente de publicación para mayo de 2013. La información de este artículo relativa al DSM-5 corresponde al borrador que la Asociación Americana de Psiquiatría propone (www.dsm5.org) y se considera información altamente fiable; sin embargo, hasta su publicación definitiva resulta necesario mantener la prudencia.

Los actuales sistemas nosológicos tienen limitaciones importantes, como, por ejemplo, que las fronteras entre los diagnósticos sean a menudo arbitrarias y haya que inferirlas subjetivamente, que la categoría 'trastornos no especificados' sea demasiado heterogénea, o que los casos subclínicos no se acomoden fácilmente a las categorías habituales, con lo que muchos síntomas subumbrales no se pueden incluir [26]. Por todo ello, el futuro DSM-5 propone aportar a la clasificación de los trastornos mentales un sistema 'dimensional' que resulte más útil para la investigación biológica y comportamental de marcadores subyacentes a varios cuadros clínicos con dimensiones semejantes [27].

El proyecto de revisión preliminar de los actuales criterios diagnósticos para las psicosis establece una categoría denominada 'espectro esquizofrénico y otros trastornos psicóticos', que incluye la esquizofrenia, el trastorno de la personalidad esquizotípico, el trastorno esquizofreniforme, el trastorno psicótico breve, el trastorno delirante y el trastorno esquizoafectivo, principalmente. El término esquizofrenia proviene del griego schizein, que significa 'dividir, escindir, hendir, romper', y phrēn, 'entendimiento, razón, mente'. Las personas con esquizofrenia pueden manifestar entre sus características diagnósticas nucleares: alucinaciones, ideas delirantes, trastornos del pensamiento, lenguaje desorganizado, y comportamiento desorganizado o catatónico. La esquizofrenia incluye siempre los síntomas psicóticos como característica definitoria; el autismo, nunca. El árbol de decisión para el diagnóstico diferencial de los trastornos psicóticos es la presencia de dichos síntomas positivos no debidos a los efectos fisiológicos directos de una enfermedad médica o a los efectos fisiológicos directos de una sustancia (droga, medicamento o tóxico).

¿Cómo se define el término 'psicótico'? La definición más restrictiva del término 'psicótico' se refiere a las ideas delirantes y a las alucinaciones manifiestas que se presentan en ausencia de conciencia de su naturaleza patológica; la menos restrictiva incluye las alucinaciones reconocidas como experiencias alucinatorias para la persona. La definición más amplia incorpora a los síntomas anteriores los síntomas positivos de la esquizofrenia: lenguaje desorganizado y comportamiento gravemente desorganizado o catatónico. Finalmente, cuando hablamos de psicosis, hablamos de una pérdida de los límites del 'sí mismo' (despersonalización) y de un grave deterioro de la capacidad para evaluar objetivamente de la realidad (desrealización).

En la actualidad, el DSM no contempla la esquizofrenia de inicio infantil como una categoría diagnóstica, pero hace referencia a ella afirmando que es muy poco común, aunque se han sido referidos casos de inicio a la edad de 4 o 5 años. En los niños, las características esenciales de la enfermedad son las mismas, pero suele ser especialmente difícil realizar el diagnóstico a estas edades, ya que también el lenguaje desorganizado se observa en diversos trastornos de inicio en la infancia; por ejemplo, en los trastornos de la comunicación y en los trastornos del espectro autista. Lo mismo sucede con respecto al comportamiento desorganizado y sus semejanzas con el trastorno por déficit de atención con hiperactividad y con el trastorno de movimientos estereotipados. Así pues, el lenguaje y el comportamiento desorganizado no deben atribuirse a la esquizofrenia sin que se hayan considerado estos otros trastornos más frecuentes en la infancia. Por otro lado, cuando sí se trata de una esquizofrenia de inicio en la infancia, las ideas delirantes y las alucinaciones suelen ser menos elaboradas que las que se observan en los adultos, y las alucinaciones visuales suelen ser más habituales [8].

Los trastornos del espectro autista en el DSM-5, en cambio, se enmarcarán dentro de los trastornos del neurodesarrollo sin subgrupos predeterminados, aunque, al igual que el espectro esquizofrénico, entendido de una manera dimensional y no categorial, como ya hicieran Wing y Gould con su propuesta de tríada comportamental [28]. Desaparecen, pues, las categorías discretas correspondientes a los trastornos generalizados del desarrollo (síndrome autista, síndrome de Asperger, síndrome de Rett, desintegrativo infantil y trastorno generalizado del desarrollo no especificado). El motivo más convincente para hablar de espectro autista se sustenta en el hecho de que las diferencias entre los supuestos subtipos no vienen determinadas por los síntomas específicos del autismo, sino por el nivel intelectual, la afectación del lenguaje y otras manifestaciones ajenas al núcleo autista. El borrador del DSM-5 reduce a dos los criterios diagnósticos: el primero, la alteración de la interacción social y la 
comunicación en distintos contextos, no explicable por un retraso general en el desarrollo (separados en el DSM-IV-TR como reciprocidad social y comunicación verbal y no verbal). El segundo, los patrones de actividades, intereses y comportamientos repetitivos y estereotipados de comportamiento, quedará formulado de manera similar; sin embargo, se añadirá dentro de este criterio la hipo o hiperreactividad a los estímulos sensoriales y el interés inusual en los aspectos sensoriales del entorno.

En su último artículo, Wing et al [29] propusieron al comité científico del DSM-5 replantear la inclusión de la alteración del 'instinto social' como un criterio diagnóstico para los TEA, entendido como la dificultad para pensar y predecir las consecuencias de las propias acciones para uno mismo y para los demás.

\section{El espectro autista y los síntomas positivos del espectro esquizofrénico}

Según el DSM-IV-TR [8], los síntomas característicos de la esquizofrenia incluyen un abanico de disfunciones cognoscitivas y emocionales que abarcan alteraciones de la percepción, del pensamiento inferencial, del lenguaje y la comunicación, de la organización comportamental, de la afectividad, la fluidez y productividad del pensamiento y el habla, de la capacidad hedónica, de la voluntad, y de la motivación y la atención. Algunos de estos síntomas se solapan claramente con los contemplados en el espectro autista. Aunque en la esquizofrenia pueden aparecer estereotipias y patrones repetitivos de comportamiento e intereses restrictivos, no configuran en ningún caso un criterio diagnóstico, lo que sí sucede en el autismo.

Los síntomas positivos de la esquizofrenia que hacen referencia a un exceso o distorsión de las funciones normales incluyen: una 'dimensión psicótica', caracterizada por distorsiones o exageraciones del pensamiento inferencial (ideas delirantes) y de la percepción (alucinaciones); y una 'dimensión de desorganización', caracterizada por el lenguaje y la comunicación (pensamiento desorganizado) y la organización comportamental (comportamiento gravemente desorganizado o catatónico).

Las ideas delirantes son creencias erróneas que acostumbran a implicar una mala interpretación de las percepciones o las experiencias. El contenido más frecuente de las ideas delirantes acostumbran a ser las ideas persecutorias -la persona se considera molestada, seguida, engañada, espiada o ridiculiza$\mathrm{da}-$, pero también pueden aparecer ideas delirantes autorreferenciales -creer que ciertos gestos, comen- tarios, parágrafos de libros, periódicos, canciones, están dirigidos a él/ella-; o temas somáticos, religiosos o grandiosos. Las ideas delirantes se clasifican como extrañas si son claramente improbables e incomprensibles y si no derivan de las experiencias cotidianas de la vida de la persona, como, por ejemplo, creer que un ser extraño le ha robado los órganos internos y los ha reemplazado por los de otra persona sin dejar cicatriz. Estas ideas delirantes extrañas expresan una pérdida de control sobre la mente o el cuerpo; por ejemplo, que los pensamientos han sido captados por alguna fuerza exterior ('robo del pensamiento'), que pensamientos externos han sido introducidos en la propia mente ('inserción del pensamiento') o que el cuerpo o los actos son controlados o manipulados por alguna fuerza exterior ('ideas delirantes de control'). Un ejemplo de idea delirante no extraña es la falsa creencia de creer que los otros hablan de uno mismo.

Las personas con autismo pueden mostrar una actitud sospechosa o de desconfianza hacia las personas o un pobre juicio social que puede confundirse con 'paranoia', pero que, en realidad, aparecen como consecuencia de sus déficits en el procesamiento de la información que provocan alteraciones en la percepción del mundo social. No suelen tener problemas para evaluar lo que es real o no; sin embargo, las dificultades para elaborar juicios sociales, la hipomentalización y los intereses y actividades restringidos, y a los que se dedican de manera obsesiva, pueden parecer paranoides sin serlo.

El hecho de no poder tener en cuenta el punto de vista del otro se manifiesta en un egocentrismo muy fuerte en las personas con TEA y una dificultad para adoptar una perspectiva alocéntrica; es decir, un punto de vista diferente al propio. 'Es probable que surja la sospecha y el recelo cuando no eres capaz, de manera automática, de tener en cuenta que los demás tienen puntos de vista diferentes del tuyo. Si tienes dificultades para darte cuenta de que otros pueden tener problemas que son incompatibles con los tuyos, entonces es fácil que sientas que ellos son personas hostiles para ti' [30]. Cuando somos capaces de mantener una perspectiva alocéntrica, somos también más hábiles para comprender e interpretar de manera adecuada las relaciones interpersonales. Preguntar al paciente '¿como estás?' es una demanda más compleja de lo que parece desde el punto de vista del desarrollo y requiere que la persona sea capaz de hacer objetiva su experiencia mental subjetiva, de comparar las emociones, los pensamientos y las percepciones del presente con la experiencia del pasado, e incluso compararlas con el conocimiento de la experiencia de los de- 
más - incluyendo a la persona que le entrevista (que es posible que no conozca de antes). La respuesta a esta pregunta es más complicada para una persona con autismo por lo que implica de reciprocidad social, de identificación de emociones y de capacidad para tomar perspectiva. Ello explica que algunas personas con TEA crean que todo el mundo debería interesarse en lo que ellos piensan y, cuando no es así, se desesperan. La frustración de no sentirse comprendidos puede evocar pensamientos e ideas fácilmente confundibles con las ideas [31].

Las alucinaciones, otro síntoma positivo de la esquizofrenia, son alteraciones de la percepción auditiva, visual, olfativa, gustativa y táctil, aunque las auditivas son las más habituales -voces conocidas o desconocidas que son percibidas como diferentes a los pensamientos de la propia persona y que acostumbran a dar mensajes peyorativos o amenazantes-. A veces, puede haber dos o más voces que conversan entre ellas o que mantienen comentarios continuos sobre los pensamientos o el comportamiento de la persona.

En ocasiones, la persona con autismo puede hablar sola o repetir escenas que ha observado con anterioridad, que pueden interpretarse como delirios o alucinaciones sin serlo. Cuando la persona con TEA adopta una personalidad imaginaria es consciente de que está 'jugando' o fantaseando y que no es una identidad real. En cambio, cuando se produce despersonalización en la esquizofrenia, la persona cree ser quien realmente no es. Es necesario, pues, diferenciar lo que es un delirio y/o una alucinación de lo que es un amigo imaginario, interactuar con un objeto como si fuese un amigo de verdad o exteriorizar pensamientos. Éstos son comportamientos muy comunes en la infancia, e incluso pueden persistir en la adolescencia [32].

Los TEA se caracterizan también por una hipero hiporreactividad a los estímulos sensoriales o un interés inusual por aspectos sensoriales del entorno; por ejemplo, aparente indiferencia al dolor/calor/frío, respuesta adversa a sonidos o texturas específicas, sentido del olfato o del tacto exacerbado, fascinación por las luces o los objetos que giran. De hecho, la propuesta de borrador del futuro DSM-5 lo incorpora claramente como criterio diagnóstico en la subárea relacionada con los patrones de comportamiento, intereses o actividades restringidas y repetitivas. Para el diagnóstico diferencial entre espectro autista y espectro esquizofrénico, es muy importante no confundir los intereses sensoriales inusuales o alteraciones de la integración sensorial en los TEA con las alucinaciones de la esquizofrenia $[33,34]$.
La literalidad a la hora de hacer uso del lenguaje por parte de las personas con autismo puede llevar también a malentendidos. Si preguntamos a una persona con autismo si oye voces, nos puede dar una respuesta afirmativa, refiriéndose de manera literal a que sí es capaz de oír voces, sin comprender que se trata de voces que quizás los demás no escuchan.

Respecto al pensamiento desorganizado, correspondiente a la 'dimensión de desorganización', dado que las inferencias sobre el pensamiento se basan en el lenguaje, el DSM-IV-TR pone el énfasis en el concepto de lenguaje desorganizado: 'perder el hilo de la conversación', saltar de un tema a otro ('descarrilamiento' o pérdida de las asociaciones); respuestas sin relación con las preguntas ('tangencialidad'); $y$, a veces, puede estar tan gravemente desorganizado que es prácticamente incomprensible ('incoherencia') [8].

El posible lenguaje desorganizado de una persona con TEA no ha de servir para el diagnóstico de la esquizofrenia, a menos que el grado de desorganización sea significativamente más importante que el esperable en una alteración de la comunicación [8].

Las personas con esquizofrenia tienen problemas con la fluidez del lenguaje, saltan de un tema a otro sin darse cuenta, juntan palabras porque son semántica o fonológicamente similares, hacen afirmaciones ilógicas, etc. [35]. En autismo, las conversaciones monotemáticas, idiosincrásicas, centradas en un tema de interés obsesivo, etc., pueden percibirse como incoherentes y desorganizadas. No obstante, la falta de cohesión en el discurso proviene de un déficit en el uso del lenguaje pragmático y funcional, tal y como socialmente está diseñado para que nos ayude a comunicarnos y a interaccionar. La pragmática es una subárea de la lingüística, estudiada por la psicología del lenguaje, que se dedica a la manera en que el contexto o la situación en la que se habla influyen en el significado de lo que se dice y cómo se dice. Cuando utilizamos el lenguaje, existen toda una serie de factores extralingüísticos que lo condicionan: el contexto sociocultural, el tipo de relación con las personas que hablamos, quién está presente o no, la información que compartimos con el interlocutor y la que no, etc. La afectación de la pragmática del lenguaje en el autismo se traduce en la comprensión literal de enunciados; el uso de un lenguaje idiosincrásico y estereotipado; la dificultad para comprender metáforas, bromas e ironías; la dificultad para comprender la fuerza ilocutiva de un enunciado; el hecho de no respetar las reglas que rigen las conversaciones -turnos de palabras, detectar el interés del otro en relación con lo que cuento, si el otro tiene prisa o 
no, si es adecuado acaparar la conversación y no dejar hablar a la otra persona, si el otro es la persona adecuada a quien dirigir el discurso que está haciendo-; el hecho de no contextualizar lo que se está explicando al interlocutor, etc. En definitiva, el problema radica en no poder inferir la información que no es explícita y que nos habilita para reconocer las claves y normas sociales que regulan la interacción social.

Solomon et al [34] investigaron el pensamiento formal en personas de entre 8 y 18 años con TEA y su relación con los síntomas del TEA, el control ejecutivo y la ansiedad. Los participantes con TEA manifestaron un pensamiento ilógico mayor y pérdida de asociaciones que los participantes del grupo control. El estudio confirma cómo el pensamiento ilógico está relacionado con aspectos de funcionamiento cognitivo y de control ejecutivo, relacionados con la capacidad para utilizar el contexto social para guiar nuestras acciones hacia un objetivo a través de diferentes áreas, una de las cuales es el lenguaje. La pérdida de asociaciones se relacionó con las alteraciones en la comunicación en autismo y con el estrés y la ansiedad. Por ello, cuando se produce una alteración en el pensamiento formal de las personas con TEA no responde a patrones de pensamientos característicos de la esquizofrenia, sino fruto de las alteraciones en el lenguaje pragmático inherente al espectro autista.

La alexitimia frecuente en pacientes con TEA puede conducir también a interpretaciones erróneas sobre lo que les rodea. Llama la atención la yuxtaposición que existe entre las manifestaciones de la alexitimia y las del autismo de alto funcionamiento en cuanto a lo siguientes aspectos:

- Alteraciones cognitivas: problemas de introspección, pobre capacidad imaginativa, pensamiento concreto y operatorio, y sensación de afecto plano y aburrido, aun cuando pueden ser personas inteligentes e intelectuales.

- Alteraciones de las relaciones interpersonales: dificultades para identificar adecuadamente sus propios sentimientos, pobre comunicación verbal de su estrés emocional a otras personas, dificultad para ver a los demás como fuente de ayuda, preferencia por estar solos y evitar a la gente o carencia de interés por las personas que le rodean, y reducción importante de intereses compartidos.

- Alteraciones del discurso y el lenguaje: dificultades en el uso de metáforas, lenguaje plano, banal y basado en hechos, casi ausencia completa de pensamientos basados en actitudes interiores, sentimientos, deseos, relato detallado y a menudo aburrido de acontecimientos y de su actuación en ellos, dificultad en la función simbólica, pobreza en el contenido de la comunicación y la prosodia y tono de voz monótono, respuestas emocionales a menudo demasiado formales y pedantes.

- Alteraciones del comportamiento no verbal: dificultades para identificar las emociones a través de los gestos faciales y de la comunicación no verbal [36].

En los casos de autismo, la alteración de las habilidades sociocomunicativas es primaria y criterio diagnóstico obligado; en cambio, en la esquizofrenia, es un síntoma secundario fruto de la sintomatología psicótica positiva primaria.

Respecto al comportamiento gravemente desorganizado en la esquizofrenia, éste se manifiesta de varias maneras: comportamiento infantiloide, agitación impredecible (gritar, insultar), alteración de comportamientos dirigidos a objetivos que pueden dificultar la realización de actividades de la vida cotidiana (ir sucio, vestir de manera poco corriente, etc.). También pueden aparecer comportamientos motrices catatónicos, que no son específicos de la esquizofrenia, los cuales provocan una disminución de la reactividad en el entorno y cursan con grado extremo de falta de atención (estupor catatónico), postura rígida y resistencia a ser movido (rigidez catatónica), resistencia activa a las órdenes o los intentos de ser movido (negativismo catatónico), asumir posturas inapropiadas o extrañas (posturas catatónicas) o una actividad motriz excesiva sin propósito ni estímulo provocador (agitación catatónica).

Las estereotipias, los movimientos repetitivos, la adhesión rígida a rutinas y egocentrismo, son bastante comunes en las personas con autismo y es tan amplia su manera de manifestarse que pueden parecer comportamientos 'locos' o excesivamente extraños. No obstante, para ayudar al diagnóstico diferencial con posibles rasgos psicóticos, se confirma el diagnóstico de autismo cuando la alteración mejora con el aprendizaje de habilidades para manejar la ansiedad de acuerdo con el desarrollo social, cuando el estrés diminuye en contextos familiares, y cuando los síntomas generalmente no mejoran con medicación antipsicótica [37]. Las personas con autismo, cuando se sienten fuertemente estresadas, muestran un marcado deterioro en el funcionamiento con comportamientos extraños, destructivos, lenguaje incoherente, gritos, autolesiones, que no forman parte de los criterios diagnósticos de los TEA, sino que entran dentro de la categoría de síntomas asociados. Esos síntomas de malestar tienden a desaparecer cuando los factores estresantes se desvanecen o se resuelven, y la persona vuelve a su nivel 
de funcionamiento previo. Esta recuperación no es común en personas que experimentan un verdadero episodio psicótico.

Las personas con TEA son especialmente vulnerables al estrés y las reacciones que puedan tener en situaciones 'idiosincrásicamente estresantes' para ellas pueden llegar a confundirse con comportamientos desorganizados o psicóticos, e incluso cumplir criterios para el trastorno psicótico breve. Esas situaciones estresantes suelen estar relacionadas con los cambios en la rutina, situaciones de sobreestimulación ambiental, u otras que son fácilmente identificables para los profesionales familiarizados con los TEA, lo cual permite interpretarlos correctamente. Un análisis funcional del comportamiento puede ayudarnos a prever y prevenir esos episodios de angustia y sufrimiento a través de técnicas de reestructuración cognitiva, de modificaciones y adaptaciones en el entorno o de anticipación a situaciones altamente estresantes para la persona.

Para finalizar este apartado relativo al espectro autista y los síntomas positivos del espectro esquizofrénico, es inevitable aludir a uno de los pocos estudios que investiga los síntomas positivos de la esquizofrenia en una muestra de 26 pacientes adultos con TEA entre 1994 y 2009 de una unidad de cuidados psiquiátricos intensivos [25]. Se compararon las características clínicas entre pacientes con un diagnóstico único de esquizofrenia y pacientes con TEA comórbido con la esquizofrenia. Se trabajó con pacientes de la unidad de cuidados psiquiátricos intensivos, porque la posibilidad de comparar pacientes con TEA con comorbilidad con la esquizofrenia y pacientes solamente con esquizofrenia son muy reducidas fuera de los hospitales. Primero se cribaron problemas de la comunicación, dificultades en el lenguaje, alteraciones de la interacción social, juegos inusuales, lentitud para adaptarse a los cambios, movimientos repetitivos con el cuerpo, comportamientos extraños o inapropiados, dificultades de aprendizaje y cognitivas, intereses restringidos, anormalidades faciales, torpeza y descoordinación motriz, obsesiones y compulsiones atípicas, e historial de diagnósticos psiquiátricos previos inconsistentes; especialmente, trastorno de la personalidad esquizoide o esquizotípica, fobia social y trastorno obsesivo-compulsivo. De los $22 \mathrm{ca}$ sos que manifestaron ideas delirantes y alucinaciones (84,6\% de los casos), 16 (72,73\%) recibieron un diagnóstico de esquizofrenia, y seis $(27,72 \%)$, de trastorno del estado de ánimo. El estudió constató que las personas adultas con TEA que acuden a servicios psiquiátricos a menudo son más diagnosticadas de psicosis, basándose en la presencia de síntomas positivos, como las ideas delirantes y las alucinaciones.

La mera presencia de dichos síntomas psicóticos subestima el diagnóstico de autismo y tienden a cobrar protagonismo la esquizofrenia y otros trastornos psicóticos, o el trastorno de la personalidad esquizoide. Esto puede tener unas implicaciones evidentemente negativas para el tratamiento y la efectividad de los resultados.

\section{El espectro autista y los síntomas negativos del espectro esquizofrénico}

Los síntomas negativos del espectro esquizofrénico reflejan una disminución o pérdida de las funciones normales en la expresión emocional (aplanamiento afectivo), la fluidez y la productividad del pensamiento y el lenguaje (alogia), y el inicio del comportamiento dirigido a un objetivo (abulia).

Conductualmente, las características que se asocian con los síntomas negativos que se describen en la esquizofrenia también están presentes en el autismo [21,38]. Lo que habría que preguntarse es si las teorías psicológicas que explican estas conductas son también comunes. Spek y Wouters [39] relacionan la presencia de síntomas negativos en el espectro autista con las dificultades para cambiar el foco de atención y, por ende, en la mayor capacidad para centrarse en los detalles, mientras que en las personas con esquizofrenia los síntomas negativos se relacionan justamente con lo contrario: con una alteración de la capacidad para focalizar.

El aplanamiento afectivo es especialmente frecuente en la esquizofrenia y se manifiesta a través de síntomas como, por ejemplo, inmovilidad y falta de respuesta en la expresión facial, contacto visual pobre, disminución del lenguaje corporal y expresividad emocional disminuida. En cambio, la falta de expresión emocional y el afecto aplanado en el autismo pueden responder a su dificultad para el uso de pautas no verbales de la comunicación, o a su falta de motivación en el mundo social e iniciativa de actuación en él. De hecho, no podemos hablar de disminución del lenguaje corporal, sino de alteración de la comunicación no verbal y gestual. Son muchos los estudios en los que queda patente que las personas con autismo parecen atender mucho menos aquellas partes del cuerpo que realmente nos informan del estado mental y emocional de la persona con la que interaccionamos y que son esenciales para la comunicación: los ojos y la boca, además de todo lo relativo a las sutilezas del lenguaje corporal o gestual. El déficit en el reconocimiento de las señales que rigen la comunicación no verbal 
será un criterio diagnóstico en el espectro autista y, en ningún caso, en el espectro esquizofrénico.

La abulia se manifiesta en la esquizofrenia a través de la dificultad para iniciar y persistir en actividades dirigidas a un fin. La persona puede permanecer quieta mucho tiempo y no mostrar interés en participar en actividades del tipo que sea. En el espectro autista, en cambio, la abulia tiene que ver con el sentido de la actividad, con la dificultad que se observa para dar sentido a la propia acción [40]. En los casos de mayor afectación se traduce en un vacío de la acción funcional y, en los de menor afectación, en una dificultad para proyectarse en el futuro. La falta de sentido de la actividad se relaciona también con la inflexibilidad cognitiva y conductual, la dificultad para aceptar cambios (invarianza ambiental) y las limitaciones en las competencias de anticipación.

Los síntomas negativos, especialmente el aplanamiento afectivo y la abulia, son más difíciles de evaluar, porque se dan en continuidad con la normalidad, son inespecíficos y pueden deberse a otros factores, como, por ejemplo, tomar medicación, trastorno del estado de ánimo, hipoestimulación ambiental, desmoralización, etc.

No se puede obviar respecto a la manifestación de síntomas negativos los correlatos y consecuencias de la coocurrencia entre autismo de alto funcionamiento y trastornos de ansiedad y del estado de ánimo [41-43]. La anhedonia social producto de la depresión y la inhibición social producto de la ansiedad podrían ser altamente confundidas con síntomas negativos relacionados con el humor deprimido, la disminución del interés o el placer en actividades, la fatiga o pérdida de energía, los sentimientos de inutilidad o culpa excesivos e inapropiados, la disminución de la habilidad para pensar/ concentrarse o indecisión, etc.

Por último, en la esquizofrenia de inicio precoz, la presencia de síntomas negativos es considerada una predictora muy valiosa para el diagnóstico, no así en el autismo. Así pues, no se recomienda la valoración de los síntomas negativos para el diagnóstico diferencial, sino de los síntomas positivos de la esquizofrenia.

Pródromo, inicio, evolución, sexo e historia familiar

Otras variables clínicas que se deben tener presentes cuando sea necesario hacer un diagnóstico diferencial más específico entre el espectro autista y el espectro esquizofrénico tienen que ver con el pródromo e inicio del trastorno, la evolución, el sexo y la historia familiar.
El término 'pródromo' deriva del griego pródromos, que literalmente significa 'predecesor de un evento'. En psiquiatría, un pródromo se refiere a los signos y síntomas precoces que preceden a las manifestaciones agudas y características de un trastorno. En las psicosis, aquellas manifestaciones clínicas que sobresalen del estado habitual de la persona y que preceden a la instauración de los síntomas psicóticos pueden yuxtaponerse a características de los trastornos generalizados del desarrollo no especificados -comportamientos ritualistas, verbalizaciones inusuales, afecto plano, aislamiento social-, lo cual, por definición, es una presentación atípica o parcial del autismo.

En un estudio con 75 niños con esquizofrenia de inicio infantil, se halló que el $25 \%$ de esos niños tuvo un diagnóstico de TEA, la mayoría de ellos no especificado. Lo que no queda claro es si el TEA no especificado puede ser un factor de riesgo para el desarrollo posterior de psicosis o si los criterios diagnósticos del TEA no especificado son lo suficientemente amplios como para abarcar presentaciones clínicas muy heterogéneas, que son consistentes tanto con los TEA como con un espectro esquizofrénico emergente [44]. Deprey y Ozonoff optan por la segunda de las interpretaciones [33].

En una revisión reciente con el objetivo de ver el posible solapamiento entre espectro autista y espectro esquizofrénico, se subraya el solapamiento clínico y genético entre los dos espectros, confirmando que en la mitad de los casos de niños con un inicio temprano de la sintomatología psicótica en grandes estudios longitudinales, éste fue precedido de un trastorno generalizado del desarrollo anteriormente a la manifestación de la psicosis [2]. Por ello, pacientes con esquizofrenia de inicio infantil pueden ser erróneamente diagnosticados de TEA debido al grave deterioramiento del funcionamiento del niño. Sin embargo, los estudios que hacen un seguimiento de niños con autismo hasta la vida adulta raras veces informan de síntomas psicóticos [23,24].

Respecto a la edad de inicio, la manifestación de un deterioro del funcionamiento del niño entre los 18 y los 24 meses de vida es muy común en niños con TEA y criterio clave para el diagnóstico de TEA (hasta los 30 meses) y no para el de esquizofrenia. En el próximo DSM-5, la necesidad de que en los TEA se produzcan retrasos o alteraciones en una de las tres áreas (interacción social, empleo comunicativo del lenguaje o juego simbólico) antes de los 3 años se sustituye por la necesidad de que los síntomas estén presentes desde la primera infancia, aunque no puedan percibirse hasta que las demandas sociales excedan la limitación de las capacidades. 
El comienzo de la esquizofrenia, en cambio, ocurre típicamente entre los últimos años de la segunda década de la vida y la mitad de la cuarta, siendo poco común el inicio anterior a la adolescencia. La edad media de inicio para el primer episodio psicótico es la mitad de los treinta en hombres y al final de los treinta en las mujeres. La esquizofrenia de inicio infantil es una forma de esquizofrenia no muy común (1 por cada 10.000 niños) y grave con un peor pronóstico [2], con una aparición de la psicosis antes de los 13 años (aunque, como se ha dicho con anterioridad, se han referido casos de inicio a la edad de 4 o 5 años) [45].

En el autismo, la edad de inicio -anterior a los 3 años- no tiene ningún significado fisiopatológico ni guarda relación con el pronóstico. En cambio, sí lo tiene en la esquizofrenia, donde las personas con un inicio a una edad más temprana suelen ser varones y tienen peor adaptación premórbida, menor nivel de estudios, más evidencia de anormalidades cerebrales estructurales, signos y síntomas negativos más sobresalientes, más evidencia de deterioro cognitivo evaluado neuropsicológicamente y peor evolución. Por el contrario, las personas con inicio de la esquizofrenia más tardío suelen ser mujeres, manifiestan una menor evidencia de anormalidades cerebrales estructurales o deterioro cognoscitivo y presentan una mejor evolución [8].

El curso y la evolución de la esquizofrenia es variable, con exacerbaciones y remisiones en algunas personas, mientras que otras permanecen crónicamente enfermas. No es habitual la remisión completa hasta el punto de volver a niveles de funcionamiento premórbido; sin embargo, las personas afectadas suelen responder bastante bien a la medicación antipsicótica. De los que no remiten, algunos mantienen un curso relativamente estable y otros muestran un empeoramiento progresivo. En el autismo, en cambio, la alteración suele ser crónica, continua.

Por lo que hace referencia a la afectación en función del sexo, las mujeres son más propensas a presentar un inicio tardío de la esquizofrenia, síntomas afectivos más acusados y un mejor pronóstico. Los estudios realizados en hospitales sugieren una mayor tasa de esquizofrenia en hombres, mientras que los realizados en población general han sugerido una prevalencia igual para ambos sexos. En el autismo, en cambio, es más significativa la prevalencia en hombres, llegándose incluso a pensar en el autismo como una expresión extrema del cerebro masculino. Esta hipótesis ya fue formulada de una manera informal por Asperger: 'la personalidad autista es una variante extrema de la inteligencia masculina. Incluso dentro de la variación normal encontra- mos las típicas diferencias de sexo en la inteligencia... En el individuo autista, el modelo masculino es extremo' [46]. Posteriormente, Baron-Cohen reprende esta hipótesis destacando que los hombres obtienen mayores puntuaciones en el cociente de espectro autista que las mujeres, pero los que tienen un síndrome de Asperger o un autismo de alto funcionamiento puntúan todavía más alto [47].

En relación con el patrón familiar, los familiares biológicos de primer grado de niños con autismo presentan un riesgo de padecer esquizofrenia y trastorno bipolar significativamente superior al de la población general $[48,49]$. De la misma manera, la manifestación de esquizofrenia en padres aumenta de una manera significativa el riesgo de que un hijo padezca autismo $[49,50]$. Los padres de niños con esquizofrenia de inicio precoz tienen un riesgo mucho mayor de padecer trastornos del espectro esquizofrénico que los padres de hijos con esquizofrenia de inicio en la vida adulta [3].

En un reciente estudio con 26 pacientes con TEA y síntomas psicóticos positivos asociados, el 19\% de los parientes de primer grado de los pacientes manifestaba síntomas positivos de la esquizofrenia; el $30 \%$ fue diagnosticado de trastorno del estado de ánimo, y un 3,8\%, de esquizofrenia [25].

\section{Conclusiones}

La complejidad del diagnóstico de los TEA y su heterogénea manifestación fenotípica pueden conducir a profesionales de la clínica a confundir algunos de los síntomas del autismo con la psicosis. Si bien los componentes etiológicos genéticos de ambos espectros están conectados, la realidad es que los criterios diagnósticos que los determinan están claramente diferenciados. Para confirmar la comorbilidad entre espectro autista y espectro esquizofrénico, es necesario tener en cuenta que la sintomatología psicótica no forma parte del diagnóstico de TEA y que aparecen cambios significativos en el funcionamiento de la persona más allá de la línea base, que pueden marcar un antes y un después.

A la pregunta ¿cómo puede el profesional discernir si las manifestaciones que presenta la persona son propias del espectro autista, del espectro esquizofrénico o fruto de la comorbilidad?, he aquí algunas de las claves:

- La valoración de la historia de desarrollo a lo largo del tiempo y de las diferentes situaciones como herramienta diagnóstica clave, lo que, en el caso de la psicología y psiquiatría en la vida adulta, suele estar subestimado. Los síntomas con- 
sistentes con los TEA han de evidenciarse antes de los 3 años de edad, mientras que los síntomas prodrómicos de las psicosis raramente aparecen antes del período preescolar y suelen desarrollarse con posterioridad, de manera gradual e insidiosa.

- Si los síntomas adicionales presentes no abarcan los criterios diagnósticos de los TEA y suponen cambios significativos en la línea base del comportamiento de la persona indicando la aparición de nuevas dificultades, o si la persona no responde efectivamente al tratamiento esperado, entonces debería contemplarse una posible comorbilidad.

- Si las alteraciones en el área de la sociocomunicación se limitan a síntomas positivos que pueden ser plausibles con el espectro esquizofrénico pero no se relacionan con alteraciones de la pragmática del lenguaje, las actividades simbólicas, la empatía, la hipomentalización, o los patrones restrictivos o estereotipados de comportamiento, y no han formado parte de la historia de desarrollo de la persona, entonces es más plausible que hablemos de espectro esquizofrénico.

- Es preferible realizar un diagnóstico diferencial entre espectro esquizofrénico y espectro autista basándonos en la presencia o no de síntomas positivos, dado que las similitudes entre algunas manifestaciones clínicas de los TEA y los síntomas negativos de la esquizofrenia pueden provocar que personas con TEA puntúen de manera significativa en relación con dichos síntomas negativos.

- Si los síntomas positivos aparecen con posterioridad al diagnóstico de TEA, podemos hablar de comorbilidad entre TEA y espectro esquizofrénico.

- Los síntomas clave para el diagnostico diferencial del espectro autista, y que en raras ocasiones se manifiestan en el espectro esquizofrénico, son los patrones repetitivos y estereotipados de comportamiento, las resistencias al cambio, los problemas de integración sensorial y las alteraciones de la comunicación no verbal.

Como ocurre con la mayoría de los trastornos psiquiátricos, la complejidad genética se complica con la complejidad fenotípica. Y aunque es cierto que existe una conexión genética entre el espectro autista y el espectro esquizofrénico, ello no explica ningún modelo en el cual una alteración causa la otra, sino que sugiere la probabilidad de que determinadas anormalidades genéticas puedan afectar vías comunes de cientos de genes que afectan a la regulación y el desarrollo neuronal. La prospectiva de la investigación tendrá que estudiar si el solapamiento genotípico entre autismo y esquizofrenia indica que ambas condiciones pueden emerger de vulnerabilidades del neurodesarrollo similares o que comparten mecanismos patogénicos. Además, plantea la cuestión de hasta qué punto comparten aspectos fenotípicos, como similitudes neuroanatómicas y manifestaciones comportamentales (síntomas negativos) y sociocognitivas. ¿Cómo puede ser que dichas similitudes conduzcan a progresiones diferentes en ambos espectros? Si a esto añadimos la inminente introducción en el DSM-5 del síndrome psicótico atenuado como un síndrome de riesgo de psicosis, la fotografía se hace todavía mucho más borrosa [51].

Aunque en ocasiones el diagnóstico diferencial entre ambos espectros no es sencillo, sí que resulta esencial para que los tratamientos médico-farmacológicos y psicoterapéuticos no resulten estériles. Es evidente la necesidad de una formación por parte de los clínicos tanto en trastornos mentales tradicionales como en trastornos del neurodesarrollo para proveer la mejor atención posible y reducir el salto que separa los servicios de psiquiatría infantojuvenil con los de adultos.

\section{Bibliografía}

1. White T, Anjum A, Schulz SC. The schizophrenia prodrome. Am J Psychiatry 2006; 163: 376-80.

2. Rapoport J, Chávez A, Greenstein D, Addington A, Gogtay N Autism spectrum disorders and childhood-onset schizophrenia: clinical and biological contributions to a relation revisited. J Am Acad Child Adolesc Psychiatry 2009; 48: 10-8.

3. Nicolson R, Brookner FB, Lenane M, Gochman P, Ingraham LJ, Egan MF, et al. Parental schizophrenia spectrum disorders in childhood-onset and adult-onset schizophrenia. Am J Psychiatry 2003; 160: 490-5.

4. Bleuler E. Demencia precoz. El grupo de las esquizofrenias. Buenos Aires: Lumen-Hormé; 1993.

5. Kolvin I. Studies in the childhood psychoses I. Diagnostic criteria and classification. Br J Psychiatry 1971; 118: 381-4.

6. Rutter M. Childhood schizophrenia reconsidered. J Autism Child Schizophr 1972; 2: 315-37.

7. American Psychiatric Association. Diagnostic and statistical manual of mental disorders, third edition, text revision. Washington DC: APA; 1980.

8. American Psychiatric Association. Diagnostic and statistical manual of mental disorders, fourth edition, text revision. Washington DC: APA; 2000.

9. Carroll LS, Owen MJ. Genetic overlap between autism, schizophrenia and bipolar disorder. Genome Med 2009; 1: 102.

10. Burbach JPH, Van der Zwaag B. Contact in genetics of autism and schizophrenia. Trends Neurosci 2009; 32: 69-72.

11. Stefansson H. Rujescu D, Cichon S, Pietiläinen OPH, Ingason A, Steinberg S, et al. Large recurrent microdeletions associated with schizophrenia. Nature 2008; 455: 232-6.

12. Voineskos AN, Lett TAP, Lerch JP, Tiwari AK, Ameis SH, Rajji TK, et al. Neurexin-1 and frontal lobe white matter: an overlapping intermediate phenotype for schizophrenia and autism spectrum disorders. PloS One 2011; 6: e20982.

13. Kirov G, Rujescu D, Ingason A, Collier DA, O’Donovan MC Owen MJ, et al. Neurexin 1 delections in schizophrenia. Schizophr Bull 2009; 35: 851-4. 
14. Glessner JT, Wang K, Cai G, Korvatska O, Kim CE, Wood S, et al. Autism genome-wide copy number variation reveals ubiquitin and neuronal genes. Nature 2009; 459: 569-73.

15. Cheung C, Yu K, Fung G, Leung M, Wong C, Li Q, et al. Autistic disorders and schizophrenia: related or remote? An anatomical likehood estimation. PloS One 2010; 5: e12233.

16. Petterson-Yeo W, Allen P, Benetti S, McGuire P, Mechelli A. Disconnectivity in schizophrenia: where are we now? Neurosci Biobehav Rev 2010; 35: 1110-24.

17. Minshew NJ, Keller TA. The nature of brain dysfunction in autism: functional brain imaging studies. Curr Opin Neurol 2010; 11: 205-13.

18. Baron-Cohen S, Ring HA, Bullmore ET, Wheelwright S, Ashwin $\mathrm{C}$. The amygdala theory of autism. Neurosci Biobehav Rev 2000; 24: 355-64.

19. Gur RE, McGrath C, Chan RM, Schroeder L, Turner T An fMRI study of facial emotion processing in patients with schizophrenia. Am J Psychiatry 2002; 159: 1992-9.

20. DeLong GR. Autism: new data suggest a new hypothesis. Neurology 1999, 52: 911-6.

21. Konstantareas MM, Hewitt T. Autistic disorder and schizophrenia: diagnostic overlaps. J Autism Dev Disord 2001; 31: 19-28.

22. Stahlbert O, Soderstrom H, Rastam M, Gillbergm C. Bipolar disorder, schizophrenia, and other psychotic disorders in adults with childhood onset $\mathrm{AD} / \mathrm{HD}$ and/or autism spectrum disorders. J Neuron Transm 2004; 111: 891-902.

23. Howlin P, Goode S, Hutton J, Rutter M. Adult outcome for children with autism. J Child Psychol Psychiatry 2004; 45: 212-29.

24. Taylor JL, Seltzer MM. Changes in the autism behavioral phenotype during the transition to adulthood. J Autism Dev Disord 2010; 40: 1431-46.

25. Raja M, Azzoni A. Autistic spectrum disorders and schizophrenia in the adult psychiatric setting: diagnosis and comorbidity. Psychiatr Danub 2010; 22: 514-21.

26. Craddock N, Jones I, Kirov G, Jones L. The Bipolar Affective Disorder Dimension Scale (BADDS). A dimensional scale for rating lifetime psychopathology in bipolar spectrum disorders. BMC Psychiatry 2004; 5: 19.

27. Maser JD, Patterson T. Spectrum concepts and DSM-IV: contrasts and implications for DSM-V. Psychiatr Clin North Am 2002; 25: 855-85.

28. Wing L, Gould J. Severe impairments of social interaction and associated abnormalities in children: epidemiology and classification. J Autism Child Schizophr 1979; 9: 11-29.

29. Wing L, Gould J, Gillberg CH. Autism spectrum disorders in the DSM-V: better or worse than the DSM-IV? Res Dev Disabil 2011; 32: 768-73.

30. Frith CD. Schizophrenia and theory of mind. Psychol Med 2004; 34: 385-9.

31. Abell F, Hare DJ. An experimental investigation of the phenomenology of delusional beliefs in people with Asperger's syndrome. Autism 2005; 9: 515-31.

32. Taylor M, Carlson M, Bayto M, Geron L, Charley C. The characteristics and correlates of fantasy in school-age children: imaginary companions, impersonation and social understanding. Dev Psychol 2004, 40: 1173-87.

33. Deprey L, Ozonoff S. Assessment of comorbid psychiatric conditions in autism spectrum disorders. In Goldstein S, Naglieri JA, Ozonoff S, eds. Assessment of autism spectrum disorders. New York: Guilford Press; 2009. p. 290-316.

34. Solomon M, Ozonoff S, Carter C, Caplan R. Formal thought disorder and the autism spectrum: relationship with symptoms, executive control, and anxiety. J Autism Dev Disord 2008; 38: 1474-84.

35. Andreasen N. Scale for the Assessment of Positive Symptoms (SAPS) and Scale for the Assessment of Negative Symptoms (SANS). In American Psychiatric Association, eds. Handbook of psychiatric measures. Washington DC: APA; 2000.

36. Paula I, Martos J, Llorente M. Alexitimia y síndrome de Asperger. Rev Neurol 2010; 50: 85-90.

37. Dossetor DR. All that glitters is not gold: misdiagnosis of psychosis in pervasive developmental disorders -a case series. Clin Child Psychol Psychiatry 2007; 12: 537-48.

38. Sheitman BB, Bodfish JW, Carmel H. Are the negative symptoms of schizophrenia consistent with an autistic spectrum illness? Schizophr Res 2004; 69: 119-20.

39. Spek AA, Wouters SGM. Autism and schizophrenia in high functioning adults: behavioral differences and overlap. Res Autism Spectrum Disord 2010; 4: 709-17.

40. Rivière A. Tratamiento y definición del espectro autista II: anticipación; flexibilidad y capacidades simbólicas. In Rivière A, Martos J, eds. El tratamiento del autismo. Nuevas perspectivas. Madrid: Apna-Imserso; 1997. p. 107-60.

41. Paula I, Martos J. Síndrome de Asperger y autismo de alto funcionamiento: comorbilidad con trastornos de ansiedad y del estado de ánimo. Rev Neurol 2009; 48: 31-4.

42. Ghaziuddin M, Ghaziuddin N, Greden J. Depression in persons with autism: implications for research and clinical care. J Autism Dev Disord 2002; 32: 299-306.

43. Gillott A, Frurniss F, Walter A. Anxiety in high-functioning children with autism. Autism 2001; 5: 277-86.

44. Sporn AL, Addington AM, Gogtay N, Ordóñez AE, Gornick M, Clasen L, et al. Pervasive developmental disorder and childhood-onset schizophrenia: comorbid disorder or a phenotypic variant of a very early onset illness? Biol Psychiatry 2004; 55: 989-94.

45. Hollis C, Rapoport J. Child and adolescent schizophrenia. In Weinberger D, Harrison P, eds. Schizophrenia. London: Blackwell; 2008.

46. Asperger H. Die 'Autistischen psychopathen' im Kindersalter. Eur Arch Psychiatry Clin Neurosci 1994; 117: 76-136.

47. Baron-Cohen S. La gran diferencia. Barcelona: Amat; 2007.

48. Ghaiziuddin M. A family history study of Asperger syndrome. J Autism Dev Disord 2005; 35: 177-82.

49. Daniels JL, Forssen U, Hultman CM, Cnattingius S, Savitz DA, Feychting M, et al. Parental psychiatric disorders associated with autism spectrum disorders in the offspring. Pediatrics 2008; 121: 1357-62.

50. Larsson HJ, Eaton WW, Madsen KM, Vestergaard M, Olesen, AV, Agerbo E, et al. Risk factors for autism: perinatal factors, parental psychiatric history and socioeconomic status. Am J Epidemiol 2005; 161: 916-25.

51. Woods SW, Walsh BC, Saksa JR, McGlashan TH. The case for including attenuated psychotic symptoms syndrome in DSM-5 as a psychosis risk syndrome. Schizophr Res 2010; 123: 199-207.

\section{Differential diagnosis between the autistic spectrum and the schizophrenic spectrum}

Introduction. The nosological distinction between the autistic spectrum and the schizophrenic spectrum is clearly defined today, despite scientific evidence of the genetic relationship between the two conditions. The overlap between the negative symptoms of schizophrenia and certain autistic manifestations, and the fact that professionals who are not familiar with autistic spectrum disorders have misguidedly attributed positive symptoms of schizophrenia in autism together highlight the importance of deciphering the keys that make it possible to reach a differential diagnosis or to evaluate the comorbidity and co-occurrence of both spectra when this is the case. 
Development. The article analyses and unravels the manifestations of autism that could be mistaken for the psychotic dimension and the disorganisation dimension corresponding to the positive symptoms of the schizophrenic spectrum. It also seeks to clarify the psychological explanations justifying the manifestation of certain negative symptoms frequently associated with autism.

Conclusions. The keys to determining whether the clinical manifestations belong to the autistic spectrum, the schizophrenic spectrum or result from comorbidity lie in the evaluation of the developmental history of the person, the prodrome and onset of the condition, its course and the presence or absence of positive symptoms of schizophrenia. Determining them will play a crucial role in helping the professional to make decisions concerning both the diagnosis and treatment.

Key words. Autistic spectrum. Comorbidity. Differential diagnosis. Nosology. Positive and negative symptoms. Schizophrenic spectrum. 